\title{
ON A CERTAIN CONVOLUTION OPERATOR FOR MEROMORPHIC FUNCTIONS
}

\author{
MING-PO CHEN AND SHIGEYOSHI OWA
}

\begin{abstract}
A bstract. A certain convolution operator $D^{\alpha} f(z)$ for meromorphic functions and two subclasses $M(\alpha)$ and $J(\alpha)$ are introduced. The object of the present paper is to derive some properties of the classes $M(\alpha)$ and $J(\alpha)$. The results of the paper are the generalizations and the improvements of the former results.
\end{abstract}

\section{Introduction}

Let $\Sigma$ denote the class of functions of the form

$$
f(z)=\frac{1}{z}+\sum_{n=0}^{\infty} a_{n} z^{n}
$$

which are regular in the annulus $E=\{z: 0<|z|<1\}$ with a simple pole at the origin with residue one there. For functions

$$
f_{j}(z)=\frac{1}{z}+\sum_{n=0}^{\infty} a_{n, j} z^{n} \quad(j=1,2)
$$

in the class $\Sigma$, we define the convolution of $f_{1}(z)$ and $f_{2}(z)$ by

$$
f_{1} * f_{2}(z)=\frac{1}{z}+\sum_{n=0}^{\infty} a_{n, 1} a_{n, 2} z^{n} .
$$

Using the convolution, we introduce the following convolution operator $D^{\alpha} f(z)$ by

$$
D^{\alpha} f(z)=\frac{1}{z(1-z)^{\alpha+1}} * f(z) \quad(\alpha>-1)
$$

Received August 30, 1992.

1991 Mathematics Subject Classification. Primary 30C45.

Key words and phrases. Convolution operator, meromophic functions. 
for $f(z) \in \Sigma$. A function $f(z) \in \Sigma$ is said to be in the class $M(\alpha)$ if it satisfies

$$
\operatorname{Re}\left\{\frac{D^{\alpha+1} f(z)}{D^{\alpha} f(z)}\right\}<\frac{\alpha+2}{\alpha+1}
$$

for some $\alpha(\alpha>-1)$ and for all $z \in U=\{z:|z|<1\}$. The class $M(\alpha)$ when $\alpha=n \in$ $N_{0}=\{0,1,2, \ldots\}$ was introduced by Ganigi and Uralegaddi [1].

Theorem $\mathbb{A}([1]) . M(n+1) \subset M(n)$ for each $n \in N_{0}$. satisfies

Further, a function $f(z) \in \Sigma$ is said to be a member of the class $J(\alpha)$ if it

$$
\operatorname{Re}\left\{\frac{\left(D^{\alpha+1} f(z)\right)^{\prime}}{\left(D^{\alpha} f(z)\right)^{\prime}}\right\}<\frac{\alpha+2}{\alpha+1}
$$

for some $\alpha(\alpha>-1)$ and for all $z \in U$. The class $J(\alpha)$ when $\alpha=n \in N_{0}$ was also studied by Uralegaddi and Ganigi [4].

Theorem B ([4]). $J(n+1) \subset J(n)$ for each $n \in N_{0}$.

In the present paper, we give the improvements of the above theorems.

\section{The Class $M(\alpha)$}

We begin with the statement of the following lemmas.

Lemma 1 ([3]). Let $\varphi(u, v)$ be a complex valued function,

$$
\varphi: D \rightarrow C, D \subset C^{2} \quad(C \text { is the complex plane })
$$

and let $u=u_{1}+i u_{2}, v=v_{1}+i v_{2}$. Suppose that the function $\varphi(u, v)$ satisfies

(i) $\varphi(u, v)$ is continuous in $D$;

(ii) $(1,0) \in D$ and $\operatorname{Re}\{\varphi(1,0)\}>0$;

( iii) for all $\left(i u_{2}, v_{1}\right) \in D$ such that $v_{1} \leq-\frac{1+u_{2}^{2}}{2}, \operatorname{Re}\left\{\varphi\left(i u_{2}, v_{1}\right)\right\} \leq 0$.

Let $p(z)=1+p_{1} z+p_{2} z^{2}+\cdots$ be regular in $U$ such that $\left(p(z), z p^{\prime}(z)\right) \in D$ for all $z \in U$. If

$$
\operatorname{Re}\left\{\varphi\left(p(z), z p^{\prime}(z)\right)\right\}>0 \quad(z \in U),
$$

then $\operatorname{Re}\{p(z)\}>0 \quad(z \in U)$.

Lemma 2 ([2]). Let $w(z)$ be regular in $U$ with $w(0)=0$. Then if $|w(z)|$ attains its maximum value on the circle $|z|=r$ at a point $z_{0}$, we have

$$
z_{0} w^{\prime}\left(z_{0}\right)=k w\left(z_{0}\right)
$$

where $k$ is real and $k \geq 1$.

Now, we derive 
Theorem 1. If $f(z) \in M(\alpha+1), \alpha>-1$ and $1<\beta(\alpha)<\frac{\alpha+2}{\alpha+1}$, then

$$
\operatorname{Re}\left\{\frac{D^{\alpha+1} f(z)}{D^{\alpha} f(z)}\right\}<\beta(\alpha) \quad(z \in U)
$$

where

$$
\beta(\alpha)=\frac{2 \alpha+3+\sqrt{(2 \alpha+3)^{2}+8(\alpha+1)}}{4(\alpha+1)}
$$

Proof. For $f(z) \in M(\alpha+1)$, we define the function $p(z)$ by

$$
\frac{D^{\alpha+1} f(z)}{D^{\alpha} f(z)}=\beta+(1-\beta) p(z)
$$

with $\beta=\beta(\alpha)$. Then $p(z)=1+p_{1} z+p_{2} z^{2}+\cdots$ is regular in $U$. With the help of the identity

$$
z\left(D^{\alpha} f(z)\right)^{\prime}=(\alpha+1) D^{\alpha+1} f(z)-(\alpha+2) D^{\alpha} f(z)
$$

(2.3) gives that

$$
\begin{aligned}
& \frac{\alpha+3}{\alpha+2}-\frac{D^{\alpha+2} f(z)}{D^{\alpha+1} f(z)} \\
= & \frac{1}{\alpha+2}\left\{(\alpha+2)-(\alpha+1)(\beta+(1-\beta) p(z))-\frac{(1-\beta) z p^{\prime}(z)}{\beta+(1-\beta) p(z)}\right\},
\end{aligned}
$$

that is, that

$$
\begin{aligned}
& \operatorname{Re}\left\{\frac{\alpha+3}{\alpha+2}-\frac{D^{\alpha+2} f(z)}{D^{\alpha+1} f(z)}\right\} \\
= & \operatorname{Re} \frac{1}{\alpha+2}\left\{(\alpha+2)-(\alpha+1)(\beta+(1-\beta) p(z))-\frac{(1-\beta) z p^{\prime}(z)}{\beta+(1-\beta) p(z)}\right\}>0 .
\end{aligned}
$$

Now, we define the function $\varphi(u, v)$ by

$$
\varphi(u, v)=\frac{1}{\alpha+2}\left\{(\alpha+2)-(\alpha+1)(\beta+(1-\beta) u)-\frac{(1-\beta) v}{\beta+(1-\beta) u}\right\},
$$

which satisfies $\operatorname{Re}\left\{\varphi\left(p(z), z p^{\prime}(z)\right)\right\}>0$ for $z \in U$. Also we see that

(i) $\varphi(u, v)$ is continous in $D=\left(C-\left\{\frac{\beta}{\beta-1}\right\}\right) \times C$;

(ii) $(1,0) \in D$ and $\operatorname{Re}\{\varphi(1,0)\}=\frac{1}{\alpha+2}>0$;

(iii) for all $\left(i u_{2}, v_{1}\right) \in D$ such that $v_{1} \leq-\frac{1+u_{2}^{2}}{2}$,

$$
\begin{aligned}
\operatorname{Re}\left\{\varphi\left(i u_{2}, v_{1}\right)\right\} & =\frac{1}{\alpha+2}\left\{(\alpha+2)-(\alpha+1) \beta-\frac{\beta(1-\beta) v_{1}}{\beta^{2}+(1-\beta)^{2} U_{2}^{2}}\right\} \\
& \leq \frac{1}{\alpha+2}\left\{(\alpha+2)-(\alpha+1) \beta+\frac{\beta(1-\beta)\left(1+u_{2}^{2}\right)}{2\left(\beta^{2}+(1-\beta)^{2} u_{2}^{2}\right)}\right\} \\
& =\frac{(1-\beta)\{3+2(\alpha+1)(1-\beta)\}}{2(\alpha+2)\left(\beta^{2}+(1-\beta)^{2} u_{2}^{2}\right)} u_{2}^{2} \leq 0
\end{aligned}
$$


because $2(\alpha+1) \beta^{2}-(2 \alpha+3) \beta-1=0, \alpha>-1$ and $1<\beta<\frac{\alpha+2}{\alpha+1}<\frac{2 \alpha+5}{2(\alpha+1)}=$ $1+\frac{3}{2(\alpha+1)}$. Thus the function $\varphi(u, v)$ satisfies the conditions in Lemma 1. Therefore we have that $\operatorname{Re}\{p(z)\}>0(z \in U)$, or, that

$$
\operatorname{Re}\left\{\frac{D^{\alpha+1} f(z)}{D^{\alpha} f(z)}\right\}<\beta=\frac{2 \alpha+3+\sqrt{(2 \alpha+3)^{2}+8(\alpha+1)}}{4(\alpha+1)} .
$$

Corollary 1. $M(\alpha+1) \subset M(\alpha)$ for $\alpha>-1$.

Proof. Noting that $1<\beta(\alpha)<\frac{\alpha+2}{\alpha+1}$, we have the corollary.

Remark 1. Theorem 1 is the improvement of Theorem A by Ganigi and Uralegaddi [1].

Taking $\alpha=0$ in Theorem 1 , we have

Corollary 2. If $f(z) \in M(1)$, Then

$$
\operatorname{Re}\left\{-\left(\frac{z f^{\prime}(z)}{f(z)}\right)\right\}>\frac{5-\sqrt{17}}{4} \quad(z \in E)
$$

that is, $f(z)$ is starlike of order $(5-\sqrt{17}) / 4$.

Applying Lemma 2, we prove

Theorem 2. If $f(z) \in \Sigma$ satisfies

$$
\operatorname{Re}\left\{\frac{D^{\alpha+1} f(z)}{D^{\alpha} f(z)}\right\}<\frac{1+2(\alpha+2)(c+1)}{2(\alpha+1)(c+1)} \quad(z \in U)
$$

for $\alpha>-1$ and $c>0$, then the function $F(z)$ defined by

$$
F(z)=\frac{c}{z^{c+1}} \int_{0}^{z} t^{c} f(t) d t
$$

is in the class $M(\alpha)$, where $F(z) \neq 0(z \in E)$.

Proof. From the definition of $F(z)$, it can be verified that

$$
z\left(D^{\alpha} F(z)\right)^{\prime}=c D^{\alpha} f(z)-(c+1) D^{\alpha} F(z) \quad(\alpha>-1) .
$$

Therefore, using (2.12) and

$$
z\left(D^{\alpha} F(z)\right)^{\prime}=(\alpha+1) D^{\alpha+1} F(z)-(\alpha+2) D^{\alpha} F(z) \quad(\alpha>-1),
$$

our condition (2.10) may be written as

$$
\operatorname{Re}\left\{\frac{(\alpha+2) \frac{D^{\alpha+2} F(z)}{D^{\alpha+1} F(z)}-(\alpha+2-c)}{(\alpha+1)-(\alpha+1-c) \frac{D^{\alpha} F(z)}{D^{\alpha+1} F(z)}}\right\}<\frac{1+2(\alpha+2)(c+1)}{2(\alpha+1)(c+1)}
$$


We define the function $w(z)$ by

$$
\frac{D^{\alpha+1} F(z)}{D^{\alpha} F(z)}=\frac{\alpha+2}{\alpha+1}-\frac{1}{\alpha+1} \frac{1-w(z)}{1+w(z)}=\frac{(\alpha+1)+(\alpha+3) w(z)}{(\alpha+1)(1+w(z))}
$$

Then $w(z)$ is regular in $U$ with $w(0)=0$. Making use of the logarithmic differentiations of both sides of (2.15), and simplifying we have

$$
\begin{aligned}
& \frac{(\alpha+2) \frac{D^{\alpha+2} F(z)}{D^{\alpha+1} F(z)}-(\alpha+2-c)}{(\alpha+1)-(\alpha+1-c) \frac{D^{\alpha} F(z)}{D^{\alpha+1} F(z)}} \\
= & \frac{(\alpha+1)+(\alpha+3) w(z)}{(\alpha+1)(1+w(z))}+\frac{2 z w^{\prime}(z)}{(\alpha+1)(1+w(z))(c+(c+2) w(z))}
\end{aligned}
$$

Suppose that there exists a point $z_{0} \in U$ such that $\max _{|z| \leq\left|z_{0}\right|}|w(z)|=\left|w\left(z_{0}\right)\right|=1$. Then, using Lemma 2, we have

$$
z_{0} w^{\prime}\left(z_{0}\right)=k w\left(z_{0}\right) \quad(k \geq 1)
$$

It follows from the above that

$$
\begin{aligned}
& \operatorname{Re}\left\{\frac{(\alpha+2) \frac{D^{\alpha+2} F\left(z_{0}\right)}{D^{\alpha+1} F\left(z_{0}\right)}-(\alpha+2-c)}{(\alpha+1)-(\alpha+1-c) \frac{D^{\alpha} F\left(z_{0}\right)}{D^{\alpha+1} F\left(z_{0}\right)}}\right\} \\
= & \operatorname{Re}\left\{\frac{\alpha+2}{\alpha+1}+\frac{2 k w\left(z_{0}\right)}{(\alpha+1)\left(1+w\left(z_{0}\right)\right)\left(c+(c+2) w\left(z_{0}\right)\right)}\right\} \\
= & \operatorname{Re}\left\{\frac{\alpha+2}{\alpha+1}+\frac{2 k w\left(z_{0}\right)\left(c+(c+2) \overline{w\left(z_{0}\right)}\right)}{(\alpha+1)\left(1+w\left(z_{0}\right)\right)\left|c+(c+2) w\left(z_{0}\right)\right|^{2}}\right\} \\
\geq & \frac{1+2(\alpha+2)(c+1)}{2(\alpha+1)(c+1)} .
\end{aligned}
$$

This contradicts our condition (2.10). Thus $|w(z)|<1$ for all $z \in U$, or

$$
\operatorname{Re}\left\{\frac{D^{\alpha+1} F(z)}{D^{\alpha} F(z)}\right\}<\frac{\alpha+2}{\alpha+1} \quad(z \in U) .
$$

Remark 2. Taking $\alpha=n \in N_{0}$ in Theorem 2, we have the result by Ganigi and Uralegaddi [1].

Further, we have

Theorem 3. If $f(z) \in M(\alpha)$, then the function $F(z)$ given by

$$
G(z)=\frac{\alpha+1}{z^{\alpha+2}} \int_{0}^{z} t^{\alpha+1} f(t) d t
$$


is in the class $M(\alpha+1)$, where $G(z) \neq 0$ for $z \in E$.

Proof. Since

$$
(\alpha+1) D^{\alpha} f(z)=(\alpha+1) D^{\alpha+1} G(z)
$$

and

$$
(\alpha+1) D^{\alpha+1} f(z)=(\alpha+2) D^{\alpha+2} G(z)-D^{\alpha+1} G(z)
$$

we see that

$$
\operatorname{Re}\left\{\frac{\alpha+2}{\alpha+1}-\frac{\alpha+2}{\alpha+1} \frac{D^{\alpha+2} G(z)}{D^{\alpha+1} G(z)}+\frac{1}{\alpha+1}\right\}=\left\{\frac{\alpha+2}{\alpha+1}-\frac{D^{\alpha+1} f(z)}{D^{\alpha} f(z)}\right\}>0
$$

for $f(z) \in M(\alpha)$. It follows from (2.21) that $G(z) \in M(\alpha+1)$.

Remark 3. Letting $\alpha=n \in N_{0}$, Theorem 3 gives the corresponding result by Ganigi and Uralegaddi [1].

\section{The Class $J(\alpha)$}

For our class $J(\alpha)$, we prove

Theorem 4. If $f(z) \in J(\alpha+1), \alpha>-1$, then

$$
\left\{\frac{\left(D^{\alpha+1} f(z)\right)^{\prime}}{\left(D^{\alpha} f(z)\right)^{\prime}}\right\}<\beta(\alpha) \quad(z \in U)
$$

where $\beta(\alpha)$ is given by (2.2).

Proof. Defining the function $p(z)$ by

$$
\frac{\left(D^{\alpha+1} f(z)\right)^{\prime}}{\left(D^{\alpha} f(z)\right)^{\prime}}=\beta+(1-\beta) p(z)
$$

with $\beta=\beta(\alpha)$, we see that $p(z)=1+p_{1} z+p_{2} z^{2}+\cdots$ is regular in $U$ and

$$
\frac{z\left(D^{\alpha+1} f(z)\right)^{\prime \prime}}{\left(D^{\alpha+1} f(z)\right)^{\prime}}-\frac{z\left(D^{\alpha} f(z)\right)^{\prime \prime}}{\left(D^{\alpha} f(z)\right)^{\prime}}=\frac{(1-\beta) z p^{\prime}(z)}{\beta+(1-\beta) p(z)} \text {. }
$$

Using that

$$
z\left(D^{\alpha} f(z)\right)^{\prime \prime}=(\alpha+1)\left(D^{\alpha+1} f(z)\right)^{\prime}-(\alpha+3)\left(D^{\alpha} f(z)\right)^{\prime}
$$

(3.3) can be written as

$$
\frac{\left(D^{\alpha+2} f(z)\right)^{\prime}}{\left.D^{\alpha+1} f(z)\right)^{\prime}}=\frac{1}{\alpha+2}\left\{1+(\alpha+1)(\beta+(1-\beta) p(z))+\frac{(1-\beta) z p^{\prime}(z)}{\beta+(1-\beta) p(z)}\right\},
$$


or

$$
\begin{aligned}
& \frac{\alpha+3}{\alpha+2}-\frac{\left(D^{\alpha+2} f(z)\right)^{\prime}}{\left(D^{\alpha+1} f(z)\right)^{\prime}} \\
= & \frac{1}{\alpha+2}\left\{(\alpha+2)-(\alpha+1)(\beta+(1-\beta) p(z))-\frac{(1-\beta) z p^{\prime}(z)}{\beta+(1-\beta) p(z)}\right\} .
\end{aligned}
$$

Therefore, using the same manner as in Theorem 1, we conclude that $\operatorname{Re}\{p(z)\}>0$ $(z \in U)$. Noting that $\beta>1,(3.2)$ gives

$$
\operatorname{Re}\left\{\frac{\left(D^{\alpha+1} f(z)\right)^{\prime}}{\left(D^{\alpha} f(z)\right)^{\prime}}\right\}<\beta=\frac{2 \alpha+3+\sqrt{(2 \alpha+3)^{2}+8(\alpha+1)}}{4(\alpha+1)}
$$

Since $1<\beta(\alpha)<\frac{\alpha+2}{\alpha+1}$, Theorem 4 gives

Corollary 3. $J(\alpha+1) \subset J(\alpha)$ for $\alpha>-1$.

Remark 4. Theorem 4 is the improvement of Theorem B by Uralegaddi and Ganigi [4].

Letting $\alpha=0$ in Theorem 4, we have

Corollary 4. If $f(z) \in J(1)$, then

$$
\operatorname{Re}\left\{-\left(1+\frac{z f^{\prime \prime}(z)}{f^{\prime}(z)}\right)\right\}>\frac{5-\sqrt{17}}{4} \quad(z \in E),
$$

or, $f(z)$ is convex of order $(5-\sqrt{17}) / 4$.

Finally, Using the smae techniques as in the proofs of Theorem 2 and Theorem 3 , we have

Theorem 5. If $f(z) \in \Sigma$ satisfies

$$
\operatorname{Re}\left\{\frac{\left(D^{\alpha+1} f(z)\right)^{\prime}}{\left(D^{\alpha} f(z)\right)^{\prime}}\right\}<\frac{1+2(\alpha+2)(c+1)}{2(\alpha+1)(c+1)} \quad(z \in U)
$$

for $\alpha>-1$ and $c>0$, then the function $F(z)$ given by (2.11) is in the class $J(\alpha)$, where $F(z) \neq 0(z \in E)$.

Remark 5. Taking $\alpha=n \in N_{0}$ in Theorem 5, we have the result by Uralegaddi and Gamigi [4].

Theorem 6. If $f(z) \in J(\alpha)$, then the function $G(z)$ defined by (2.18) is in the class $J(\alpha+1)$, where $G(z) \neq 0$ for $z \in E$.

Remark 6. For $\alpha=n \in N_{0}$, Theorem 6 gives the corresponding result due to Uralegaddi and Ganigi [4]. 


\section{Acknowledgment}

This paper of the authors was completed at Institute of Mathematics, Academia Sinica, Taipei, Taiwan while the second author was visiting from Kinki University, Higashi-Osaka, Osaka 577, Japan. The work of Ming-Po Chen was supported by the National Science Council of R.O.C. under Grant NSC-82-0208-M-1-145.

\section{References}

[1] M.D. Ganigi and B.A. Uralegaddi, "New criteria for meromorphic univalent functions," Bull. Math. Soc. Sci. Math. R. S. Roum. 33(1989), 9-13.

[2] I.S. Jack, "Functions starlike and convex of order $\alpha$," J. London Math. Soc. (2)3(1971), 469-474.

[3] S.S. Miller and P.T. Mocanu, "Second order differential inequalities in the complex plane," J. Math. Anal. Appl. 65(1978), 289-305.

[4] B.A. Uralegaddi and M.D. Ganigi, "A new criterion for meromorphic convex functions," Tamkang J. Math. 19(1988), 43-48.

Institute of Mathematics Academia Sinica, Taipei, Taiwan, Republic of China.

Department of Mathematics, Kinki University, Higashi-Osaka, Osaka 577, Japan. 